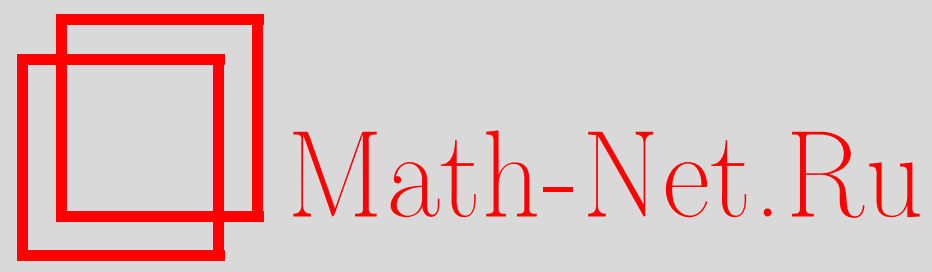

А. Г. Токарев, Обобщение формулы Березина на некоммутативный случай, Матем. заметки, 2001, том 69, выпуск $2,295-302$

DOI: https://doi.org/10.4213/mzm504

Использование Общероссийского математического портала Math-Net.Ru подразумевает, что вы прочитали и согласны с пользовательским соглашением http://www.mathnet.ru/rus/agreement

Параметры загрузки:

IP: 54.172 .240 .79

26 апреля 2023 г., 10:54:00

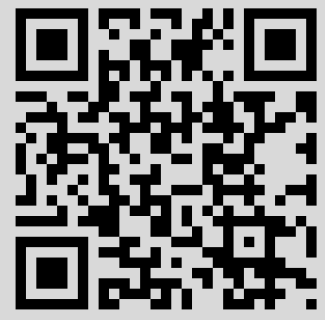




\section{ОБОБЩЕНИЕ ФОРМУЛЫ БЕРЕЗИНА НА НЕКОММУТАТИВНЫЙ СЛУЧАЙ}

\section{А. Г. Токарев}

В пространствах Фока с помощью функциональных интегралов построены представления экспонент от операторов, являющихся квадратичными функциями от операторов рождения и уничтожения с некоммутирующими коэффициентами (удовлетворяющими некоторым ограничениям аналитического характера).

Библиограффия: 7 названий.

В работе с помощью функциональных интегралов построена сильно непрерьвная полугруппа унитарных операторов с генератором $i \bar{A}$, где $\bar{A}$ - самосопряженный оператор в $L^{2}\left(\mathbb{R}^{l}\right) \otimes \mathscr{F}(L)$ вида

$$
\bar{A}=I \otimes \widehat{a}^{\dagger} E \widehat{a}+\widehat{K} \otimes \widehat{a}^{\dagger} C \widehat{a}+\widehat{R}^{\dagger} \otimes \widehat{a}\left(f^{*}\right)+\widehat{R} \otimes \widehat{a}^{\dagger}(f)+\widehat{H}_{0} \otimes I .
$$

Здесь $L$ - гильбертово пространство с инволюцией $*, \mathscr{F}(L)$ - симметризованное пространство Фока над $L, f \in L, \widehat{a}^{\dagger}(f), \widehat{a}\left(f^{*}\right)$ - операторы рождения и уничтожения, $C, E-$ самосопряженные операторы в $L$ и $C$ - ограниченньй оператор; $\widehat{K}, \widehat{R}, \widehat{H}_{0}-$ псевдодифференциальные операторы в $L^{2}\left(\mathbb{R}^{l}\right)$, символы которых являются преобразованиями $\Phi$ урье мер ограниченной вариации, символ † обозначает эрмитово сопряжение и $I$ - тождественньй оператор в соответствуюшем пространстве. Дано описание таких полугрупп как в представлении $\Phi$ ока, так и в представлении Баргмана-Фока.

Результаты этой статьи по существу являются некоммутативными аналогами формулы Березина $[1$, гл. III, $\S 6$, теорема II] и позволяют в рамках описанного вьше подхода устанавливать связи между квантовыми стохастическими дифференциальными уравнениями и унитарньми однопараметрическими полугрупами в некоммутативном случае.

1. Некоторые обозначения. Терминология и обозначения из [2] и [3] используются обычно без объяснений. Все встречающиеся здесь банаховы пространства являются сепарабельньми. Пусть $\mathbb{R}_{+}=[0, \infty)$.

Пусть $Z_{1}, Z_{2}$ - банаховы пространства. Пусть $\mathscr{L}\left(Z_{1}, Z_{2}\right)$ - пространство ограниченных линейньг отображений из $Z_{1}$ в $Z_{2}$ со стандартной нормой $\|\cdot\| \mathscr{L}, \mathscr{L}(Z)=\mathscr{L}(Z, Z)$.

Пусть $\mathscr{B}_{X}=\mathscr{B}(X)$ - борелевская $\sigma$-алгебра подмножеств топологического пространства $X$. Во всех банаховых пространствах и их борелевских подмножествах фиксируются борелевские $\sigma$-алгебры.

Работа выполнена при финансовой поддержке Российского фонда фундаментальных исследований, грант № 99-01-012012. 
Пусть $\left(X, \Sigma_{X}\right)$ - измеримое пространство. Мерой на $X$ со значениями в $Z$ будем назьвать счетно-аддитивную функцию $\mu: \Sigma_{X} \rightarrow Z$, обладающую конечной полной вариацией $\operatorname{var}(\mu)$. Вариация меры $\mu$ обозначается символом $|\mu|$. Таким образом, вариация это положительная мера на $X$ и $|\mu|(X)=\operatorname{var}(\mu)$.

Символом $L^{2}(X, \mu, Z)$ обозначим банахово пространство классов эквивалентности совпадаюших почти всюду относительно меры $\mu \mu$-измеримых отображений $\varphi: X \rightarrow Z$ таких, что

$$
\int_{X}\|\varphi(q)\|_{Z}^{2} d q<\infty
$$

с нормой $\|\cdot\|$, определяемой равенством

$$
\|\varphi\|^{2}=\int_{X}\|\varphi(x)\|_{Z}^{2} d x
$$

Если $L$ - комплексное гильбертово пространство, то $L^{2}(X, \mu, L)$ - гильбертово пространство со скалярным произведением

$$
\left\langle\varphi_{1}, \varphi_{2}\right\rangle=\int_{X}\left\langle\varphi_{1}(x), \varphi_{2}(x)\right\rangle_{L} \mu(d x)
$$

При этом существует канонический изоморфизм между пространством $L^{2}(X, \mu, L)$ и гильбертовым тензорным произведением пространств $L^{2}(X, \mu, \mathbb{C})$ и $L$. В случае, когда $X=\mathbb{R}^{l}$ и $\mu$-мера Лебега, положим $L^{2}\left(\mathbb{R}^{l}, Z\right)=L^{2}\left(\mathbb{R}^{l}, \mu, Z\right)$ и $L^{2}\left(\mathbb{R}^{l}\right)=L^{2}\left(\mathbb{R}^{l}, \mu, \mathbb{C}\right)$.

Псевдодифференциальньй оператор $\widehat{H}: L^{2}\left(\mathbb{R}^{n}\right) \rightarrow L^{2}\left(\mathbb{R}^{n}\right)$ с $q p$-символом $H: \mathbb{R}^{n} \times \mathbb{R}^{n}$ $\rightarrow \mathbb{C}$, удовлетворяющим определенным условиям, определяется с помощью выражения

$$
(\widehat{H} \varphi)(q)=(2 \pi)^{-n} \int_{\mathbb{R}^{n}} \int_{\mathbb{R}^{n}} H(q, p) e^{i p\left(q^{\prime}-q\right)} \varphi\left(q^{\prime}\right) d q^{\prime} d p
$$

Нетрудно показать, что если

$$
H(q, p)=\int_{\mathbb{R}^{n} \times \mathbb{R}^{n}} e^{i q y+i p x} \mu(d y, d x)
$$

- преобразование Фурье меры $\mu: \mathscr{B}\left(\mathbb{R}^{n} \times \mathbb{R}^{n}\right) \rightarrow \mathbb{C}$, то псевдодифференциальньй оператор $\widehat{H}$ с $q p$-символом $H$ определен и для $\varphi \in L^{2}\left(\mathbb{R}^{n}\right)$ справедливо равенство

$$
(\widehat{H} \varphi)(\cdot)=\int_{\mathbb{R}^{n} \times \mathbb{R}^{n}} e^{i\langle\cdot, y\rangle} \varphi(\cdot-x) \mu(d y, d x) .
$$

Поскольку символы всех встречающихся здесь псевдодифференциальных операторов являются преобразованиями Фурье ограниченных мер, мы примем эту формулу в качестве определения псевдодифференциального оператора $\widehat{H}$ с $q p$-символом $H$. 
2. Интегралы по векторнозначным мерам. Утверждения этого пункта являются обобщениями теорем классической теории меры и могут быть доказаны аналогичными методами.

Пусть $Z, T, L$ - банаховы пространства. Пусть $\left(X, \Sigma_{X}\right)$ - измеримое пространство и $\mu: \Sigma_{X} \rightarrow Z$ - мера на $X$. Определим интеграл $\int_{X} f(x) \mu(d x)$ отображения $f: X \rightarrow T$ по мере $\mu$ при фиксированной непрерьвной билинейной форме $p: T \times Z \rightarrow L$.

Предположим сначала, что $f: X \rightarrow T$ - простая функция, т.е. $f=\sum_{k=1}^{n} b_{k} \chi_{E_{k}}$, где $\chi_{E_{k}}$ - характеристическая функция множества $E_{k} \in \Sigma_{X}$ и $b_{k} \in T$. Определим интеграл $f$ по мере $\mu$ равенством

$$
\int_{X} f(x) \mu(d x)=\sum_{k=1}^{n} p\left(b_{k}, \mu\left(E_{k}\right)\right)
$$

Произвольную функцию $f$ назовем интегрируемой по мере $\mu$, если существует последовательность простых функций $f_{m}: X \rightarrow T$ такая, что $f_{m}(x) \rightarrow f(x)$ почти всюду относительно меры $|\mu|$ и для всякого $\varepsilon>0$ найдется $N=N(\varepsilon)$ такое, что

$$
\int\left\|f_{m}(x)-f_{k}(x)\right\|_{T}|\mu|(d x)<\varepsilon
$$

для всех $m, k>N$. В этом случае последовательность $\int_{X} f_{m}(x) \mu(d x)$ фундаментальна в $L$, ее предел назовем интегралом $f$ по $\mu$ при фиксированной форме $p$ и обозначим $\int_{X} f(x) \mu(d x)$.

В дальнейшем при интегрировании мы часто будем использовать описанную конструкцию. При этом всегда будет ясно, какая конкретно используется форма $p$, хотя в записи интеграла $\int_{X} f(x) \mu(d x)$ мы ее указьвать не будем.

3. Функциональные интегралы. Всюду ниже $P=Q=\mathbb{R}^{l}$. Здесь эти обозначения используются лишь для удобства, хотя пространствам $Q$ и $P$ можно придать смысл конфигурационного и импульсного пространств соответственно. Пусть

$$
\langle q, p\rangle=q p=p q=\sum_{k=1}^{l} p_{k} q_{k}
$$

для $p=\left(p_{1}, \ldots, p_{l}\right) \in P, q=\left(q_{1}, \ldots, q_{l}\right) \in Q$.

Через $F_{P}$ и $F_{Q}$ обозначим пространства мер ограниченной вариации на $\mathbb{R}_{+}=[0, \infty)$ со значениями в $P$ и $Q$ соответственно. Пусть $F=F_{P} \times F_{Q}$. Элементы $F$ будем обозначать $y=\left(y_{P}, y_{Q}\right), y_{P} \in F_{P}, y_{Q} \in F_{Q}$.

Вещественное линейное пространство всех ограниченных кусочно-непрерывных, непрерывных справа функций на $[0, \infty)$ со значениями в $Q$ обозначим через $F_{Q}^{\prime}$, а со значениями в $P$ - через $F_{P}^{\prime}$. Положим $F^{\prime}=F_{Q}^{\prime} \times F_{P}^{\prime}$. Элементы $F^{\prime}$ будем обозначать $x=\left(x_{Q}, x_{P}\right), x_{Q} \in F_{Q}^{\prime}, x_{P} \in F_{P}^{\prime}$.

Определим билинейную форму $\langle\cdot, \cdot\rangle: F^{\prime} \times F \rightarrow \mathbb{R}$ равенством

$$
\left\langle\left(x_{Q}, x_{P}\right),\left(y_{P}, y_{Q}\right)\right\rangle=\int_{0}^{\infty} x_{Q}(\tau) y_{P}(d \tau)+\int_{0}^{\infty} x_{P}(\tau) y_{Q}(d \tau)
$$


Обозначим через $\Sigma_{F} \sigma$-алгебру подмножеств $F$, порожденную функционалами $\langle x, \cdot\rangle$, $x \in F^{\prime}$.

Определим отображение

$$
g: F \rightarrow \mathbb{R}, \quad g:\left(y_{P}, y_{Q}\right) \mapsto \int_{0}^{\infty} y_{Q}(\tau, \infty) y_{P}(d \tau)
$$

Пусть $T, L$ - комплексные банаховы пространства. Пусть $f: F^{\prime} \rightarrow \mathscr{L}(T, L)$ - преобразование Фурье меры $\mu: \Sigma_{F} \rightarrow \mathscr{L}(T, L)$. Зафиксируем непрерывное билинейное отображение $p: L^{2}\left(\mathbb{R}^{l}, T\right) \times \mathscr{L}(T, L) \rightarrow L^{2}\left(\mathbb{R}^{l}, L\right), p:(\psi, A) \mapsto A \psi$, где $(A \psi)(q)=A \psi(q)$, $q \in \mathbb{R}^{l}$. Тогда для $\varphi \in L^{2}\left(\mathbb{R}^{l}, T\right)$ отображение

$\Psi_{\varphi}: F \rightarrow L^{2}\left(\mathbb{R}^{l}, T\right), \quad \Psi_{\varphi}:\left(y_{P}, y_{Q}\right) \mapsto \exp \left(i\left\langle\cdot, y_{P}[0, \infty)\right\rangle-i g\left(y_{P}, y_{Q}\right)\right) \varphi\left(\cdot-y_{Q}[0, \infty)\right)$

интегрируемо по мере $\mu$. Определим оператор $I_{g}(f): L^{2}\left(\mathbb{R}^{l}, T\right) \rightarrow L^{2}\left(\mathbb{R}^{l}, L\right)$ равенством

$$
I_{g}(f) \varphi=\int_{F} \Psi_{\varphi}(y) \mu(d y)=\int_{F} e^{i\left\langle\cdot, y_{P}[0, \infty)\right\rangle-i g\left(y_{P}, y_{Q}\right)} \varphi\left(\cdot-y_{Q}[0, \infty)\right) \mu\left(d y_{P}, d y_{Q}\right),
$$

где $\varphi \in L^{2}\left(\mathbb{R}^{l}, T\right)$. Отметим, что $\left\|I_{g}(f)\right\| \leqslant \operatorname{var}(\mu)$.

Между пространствами $L$ и $\mathscr{L}(\mathbb{C}, L)$ существует естественньй изометрический изоморфизм $j: L \rightarrow \mathscr{L}(\mathbb{C}, L), j: b \mapsto \widehat{b}$, где $\widehat{b} \in \mathscr{L}(\mathbb{C}, L), \widehat{b}: z \mapsto b z$. В силу этого и приведенной вьше конструкции каждой функции $f: F^{\prime} \rightarrow L$, являющейся преобразованием Фурье меры $\mu: \Sigma_{F} \rightarrow L$, поставим в соответствие оператор $I_{g}(f): L^{2}\left(\mathbb{R}^{l}\right) \rightarrow L^{2}\left(\mathbb{R}^{l}, L\right)$ по формуле (1).

Заметим, что оператор $I_{g}(f) \varphi$ можно представить в виде

$$
\left(I_{g}(f) \varphi\right)(q)=\int_{F^{\prime}} f\left(x_{Q}+q, x_{P}\right) \varphi\left(x_{Q}(0)+q\right) \Phi_{g}\left(d x_{Q}, d x_{P}\right)
$$

где интеграл в правой части можно понимать как функциональньй интеграл по траекториям в фазовом пространстве, определенньй с помощью равенства Парсеваля (ср. [4, гл. $3, \S 2],[5],[6])$.

Используя $[2$, гл. III, п. 5 , следствие 9], можно показать, что любая мера на $F$ однозначно определяется своим преобразованием Фурье. Поэтому функция $f$ однозначно определяет некоторьй оператор $I_{g}(f)$ по формуле (1).

4. Основные меры, их преобразования Фурье и свойства. Далее мы будем использовать следующую конструкцию. Пусть $\left(\Omega, \Sigma_{\Omega}\right)$ - измеримое пространство и $\mu: \Sigma_{\Omega} \rightarrow \mathbb{C}$ - скалярная мера на $\Omega$. Пусть $L-$ сепарабельное гильбертово пространство и отображение $\rho: \Omega \rightarrow \mathscr{L}(L)$ удовлетворяет следующим условиям. Функция $\|\rho(\cdot)\|_{\mathscr{L}}$ измерима и

$$
\int_{\Omega}\|\rho\| \mathscr{L} d|\mu|<\infty
$$

отображение $\rho(\cdot) b: \Omega \rightarrow L, \rho(\cdot) b: \omega \mapsto \rho(\omega) b$ измеримо по Бохнеру для всех $b \in L$. Для $M \in \Sigma_{\Omega}$ определим интеграл $\int_{M} \rho(\omega) \mu(d \omega)$ как ограниченный линейньй оператор в $L$ такой, что

$$
\left(\int_{M} \rho d \mu\right)(b)=\int_{M} \rho(\omega) b \mu(d \omega)
$$


Это определение корректно, так как

$$
\left\|\int_{M} \rho(\omega) b \mu(d \omega)\right\|_{L} \leqslant \int_{M}\|\rho\|_{\mathscr{L}} d|\mu| \cdot\|b\|_{L}
$$

и, следовательно,

$$
\left\|\int_{M} \rho d \mu\right\|_{\mathscr{L}} \leqslant \int_{M}\|\rho\|_{\mathscr{L}} d|\mu| .
$$

Отсюда вытекает, что функция множества $\rho \mu: \Sigma_{\Omega} \rightarrow \mathscr{L}(L), \rho \mu: M \mapsto \int_{M} \rho d \mu$ счетно аддитивна и

$$
\operatorname{var}(\rho \mu) \leqslant \int_{\Omega}\|\rho\| \mathscr{L} d|\mu|
$$

Поэтому $\rho \mu$-мера. Ясно, что если $\rho(\cdot)$ - измеримоепо Бохнеру отображение, то $\int_{M} \rho d \mu$ совпадает с интегралом Бохнера функции $\rho$.

Пусть $\lambda(d r)$ - мера Лебега в $\mathbb{R}^{n}$ и $\lambda_{M}(d r)$ - сужение $\lambda$ на ограниченное борелевское множество $M \subset \mathbb{R}_{+}^{n}$. Для $r \geqslant 0$ и комплексной борелевской меры $\mu$ на $P \times Q$ обозначим через $\mu_{r}$ образ меры $\mu$ при отображении $j_{r}: P \times Q \rightarrow F, j_{r}:(p, q) \mapsto(p, q) \delta_{r}$. Определим меру $\int_{0}^{t} \mu_{\tau} d \tau: \Sigma_{F} \rightarrow \mathbb{C}$ как образ меры $\lambda_{[0, t]} \times \mu$ при отображении $[0, t] \times P \times Q \rightarrow F$, $(r, p, q) \mapsto(p, q) \delta_{r}$. Пусть $\rho: \mathbb{R}_{+}^{n} \rightarrow \mathscr{L}(L)$ - ограниченное отображение такое, что отображения $\|\rho(\cdot)\| \mathscr{L}$ и $\rho(\cdot) b, b \in L$, измеримы по Бохнеру. Для комплексных борелевских мер $\mu^{1}, \ldots, \mu^{n}$ на $P \times Q$ и ограниченного борелевского множества $M \subset \mathbb{R}_{+}^{n}$ определим меру $\varkappa: \Sigma_{F} \rightarrow \mathscr{L}(L)$,

$$
\varkappa=\int \cdots \int_{M} \rho\left(r_{1}, \ldots, r_{n}\right) \mu_{r_{1}}^{1} * \cdots * \mu_{r_{n}}^{n} d^{n} r
$$

как образ меры $\rho \lambda_{M} \times \mu^{1} \times \cdots \times \mu^{n}$ при отображении $\left.\pi_{n}\right|_{M \times(P \times Q)^{n}, \text { которое является }}$ сужением на множество $M \times(P \times Q)^{n}$ измеримого отображения $\pi_{n}: \mathbb{R}_{+}^{n} \times(P \times Q)^{n} \rightarrow F$, $\pi_{n}:\left(r_{1} \ldots, r_{n},\left(p_{1}, q_{1}\right), \ldots,\left(p_{n}, q_{n}\right)\right) \mapsto\left(p_{1}, q_{1}\right) \delta_{r_{1}}+\cdots+\left(p_{n}, q_{n}\right) \delta_{r_{n}}$. Из определения вытекает, что

$$
\begin{aligned}
\operatorname{var}(\varkappa) & \leqslant \operatorname{var}\left(\mu^{1}\right) \cdots \operatorname{var}\left(\mu^{n}\right) \int_{M}\|\rho(r)\|_{\mathscr{L}} \lambda(d r) \\
& \leqslant \operatorname{var}\left(\mu^{1}\right) \cdots \operatorname{var}\left(\mu^{n}\right) \lambda(M) \sup _{r_{k} \geqslant 0}\left\|\rho\left(r_{1}, \ldots, r_{n}\right)\right\|_{\mathscr{L}} .
\end{aligned}
$$

Пусть $C \in \mathscr{L}(L)$ и $E$ - самосопряженньй, возможно неограниченный оператор в $L$. Определим ограниченные отображения

$$
\rho_{n}: \mathbb{R}_{+}^{n} \rightarrow \mathscr{L}(L), \quad \rho_{n}:\left(r_{1}, \ldots, r_{n}\right) \mapsto e^{-i r_{n} E} C e^{i r_{n} E} \cdots e^{-i r_{1} E} C e^{i r_{1} E}
$$

для $n \in \mathbb{N}$. Заметим, что для каждого $b \in L$ отображения $\rho_{n}(\cdot) b: \mathbb{R}_{+}^{n} \rightarrow L$ непрерывны. Пусть $\mu, \nu, \nu^{\dagger}, \theta$ - комплексные борелевские меры ограниченной вариации на $P \times Q$. 
Пусть $t \geqslant 0, \delta_{0}-$ мера Дирака на $F$, сосредоточенная в точке 0, и $I$ - тождественньй оператор в $L$. Определим четыре операторнозначные меры:

$$
\begin{aligned}
& \mu_{s, t}=e^{i t E}\left(I \delta_{0}+\sum_{n=1}^{\infty} i^{n} \int \cdots \int_{s \leqslant r_{1} \leqslant \cdots \leqslant r_{n} \leqslant t} \rho_{n}(r) \mu_{r_{1}} * \cdots * \mu_{r_{n}} d^{n} r\right) e^{-i s E} \\
& \quad 0 \leqslant s \leqslant t<\infty, \\
& \int_{0}^{t} \mu_{0, \tau} * \nu_{\tau}^{\dagger} d \tau=\int_{0}^{t} e^{i \tau E} \nu_{\tau}^{\dagger} d \tau \\
& +\sum_{n=1}^{\infty} i^{n} \int_{0}^{t} d \tau \int^{t} \ldots \int_{0 \leqslant r_{1} \leqslant \cdots \leqslant r_{n} \leqslant \tau} e^{i \tau E} \rho_{n}(r) \mu_{r_{1}} * \cdots * \mu_{r_{n}} * \nu_{\tau}^{\dagger} d^{n} r \\
& \int_{0}^{t} \nu_{\sigma} * \mu_{\sigma, t} d \sigma=\int_{0}^{t} e^{i(t-\sigma) E} \nu_{\sigma} d \sigma \\
& +\sum_{n=1}^{\infty} i^{n} \int_{0}^{t} d \sigma \int_{\cdots} \int_{\sigma \leqslant r_{1} \leqslant \cdots \leqslant r_{n} \leqslant t} e^{i t E} \rho_{n}(r) e^{-i \sigma E} \nu_{\sigma} * \mu_{r_{1}} * \cdots * \mu_{r_{n}} d^{n} r \\
& \int_{0}^{t} d \tau \int_{0}^{\tau} d \sigma \nu_{\sigma} * \mu_{\sigma, \tau} * \nu_{\tau}^{\dagger}=\int_{0}^{t} d \tau \int_{0}^{\tau} d \sigma e^{i(\tau-\sigma) E} \nu_{\sigma} * \nu_{\tau}^{\dagger}+\sum_{n=1}^{\infty} i^{n} \int_{0}^{t} d \tau \int_{0}^{\tau} d \sigma \\
& \quad \times \int^{i} \ldots \int_{\sigma \leqslant r_{1} \leqslant \cdots \leqslant r_{n} \leqslant \tau} e^{i \tau E} \rho_{n}(r) e^{-i \sigma E} \nu_{\sigma} * \mu_{r_{1}} * \cdots * \mu_{r_{n}} * \nu_{\tau}^{\dagger} d^{n} r
\end{aligned}
$$

В формулах (2)-(5) правые части являются определением левых, а символы интегралов в левых частях формул используются лишь для обозначения.

Так как вариация $n$-го члена ряда в (2) не превосходит $\|C\|_{\mathscr{L}}^{n} \operatorname{var}(\mu)^{n} / n !$, то ряд в $(2)$ сходится по вариации. То же справедливо для мер (3)-(5).

Пусть $K, R, R^{\dagger}, H_{0}$ - преобразования $\Phi$ урье мер $\mu, \nu, \nu^{\dagger}, \theta: \mathscr{B}(P \times Q) \rightarrow \mathbb{C}$ на $P \times Q$ соответственно. Пусть $W_{s}^{t}, f_{1, t}, f_{2, t}, f_{3, t}: F^{\prime} \rightarrow \mathscr{L}(L)$ - преобразования Фурье мер $\mu_{s, t}$, $(3),(4),(5)$ соответственно и отображение $f_{4, t}: F^{\prime} \rightarrow \mathbb{C}$ - преобразование Фурье меры $\int_{0}^{t} \theta_{\tau} d \tau$. Тогда можно доказать, что

$$
\begin{gathered}
f_{1, t}(x)=\int_{0}^{t} R^{\dagger}(x(\tau)) W_{0}^{\tau}(x) d \tau, \quad f_{2, t}(x)=\int_{0}^{t} W_{\sigma}^{t}(x) R(x(\sigma)) d \sigma \\
f_{3, t}(x)=\int_{0}^{t} d \tau \int_{0}^{\tau} d \sigma R^{\dagger}(x(\tau)) W_{\sigma}^{\tau}(x) R(x(\sigma)), \quad f_{4, t}(x)=\int_{0}^{t} H_{0}(x(\tau)) d \tau
\end{gathered}
$$

для $x=\left(x_{Q}, x_{P}\right) \in F^{\prime}$.

5. Основные понятия метода вторичного квантования. Пусть $L$ - сепарабельное комплексное гильбертово пространство со скалярным произведением $\langle\cdot, \cdot\rangle_{L}$, антилинейным по второму аргументу. Пусть $*: L \rightarrow L-$ инволюция в $L$, т.е. антилинейное отображение такое, что $\left\langle f_{1}^{*}, f_{2}^{*}\right\rangle_{L}=\left\langle f_{2}, f_{1}\right\rangle_{L}$ и $\left(f_{1}^{*}\right)^{*}=f_{1}$ для всех $f_{1}, f_{2} \in L$. Определим билинейное отображение $L \times L \rightarrow \mathbb{C}$ так, что $f_{1} f_{2}=\left\langle f_{1}, f_{2}^{*}\right\rangle_{L}$. Для $C \in \mathscr{L}(L)$ определим правое действие $C$ на вектор $f \in L: f C=\left(C^{\dagger} f^{*}\right)^{*}$, где $C^{\dagger}$ - оператор, эрмитово сопряженный к $C$. Тогда $f_{1}\left(C f_{2}\right)=\left(f_{1} C\right) f_{2}=f_{1} C f_{2}$. 
Пусть $\mathscr{F}_{n}(L)(n \in \mathbb{N})$ - симметризованное тензорное произведение $n$ экземпляров пространства $L$ и $\mathscr{F}_{0}(L)=\mathbb{C}$. Пусть $\mathscr{F}(L)=\bigoplus_{n=0}^{\infty} \mathscr{F}_{n}(L)$ - симметричное пространство Фока над $L$. Для $a \in L$ обозначим через $e(a)=\left(a^{\otimes n} / \sqrt{n !}\right) \in \mathscr{F}(L)$ экспоненциальньй вектор, соответствующий $a$.

Будем отождествлять пространства $L^{2}\left(\mathbb{R}^{l}\right) \otimes \mathscr{F}(L)$ и $\bigoplus_{n=0}^{\infty} L^{2}\left(\mathbb{R}^{l}\right) \otimes \mathscr{F}_{n}(L)$. В силу этого $n$-ю компоненту вектора $\Phi \in L^{2}\left(\mathbb{R}^{l}\right) \otimes \mathscr{F}(L)$ в $\bigoplus_{n=0}^{\infty} L^{2}\left(\mathbb{R}^{l}\right) \otimes \mathscr{F}_{n}(L)$ будем обозначать через $(\Phi)_{n} \in L^{2}\left(\mathbb{R}^{l}\right) \otimes \mathscr{F}_{n}(L)$.

Пусть $E$ - самосопряженньй оператор в $L$. Самосопряженньй оператор $\widehat{a}^{\dagger} E \widehat{a}$ в $\mathscr{F}(L)$ определяется тем, что в $\mathscr{F}_{n}(L)$ он равен $\sum_{k=1}^{n} I^{\otimes(k-1)} \otimes E \otimes I^{\otimes(n-k)}$ для $n \neq 0$ и 0 в $\mathscr{F}_{0}(L)$, где $I$ - тождественный оператор.

Пусть $\widehat{K}, \widehat{R}, \widehat{H}_{0} \in \mathscr{L}\left(L^{2}\left(\mathbb{R}^{l}\right)\right), \widehat{K}$ - самосопряженный оператор и $\widehat{R}^{\dagger}$ - оператор, эрмитово сопряженный к $\widehat{R}$. Пусть $E$ и $C$ - самосопряженные операторы в $L, C \in \mathscr{L}(L)$ и $f \in L$. В пространстве $L^{2}\left(\mathbb{R}^{l}\right) \otimes \mathscr{F}(L)$ определим оператор

$$
\bar{A}=I \otimes \widehat{a}^{\dagger} E \widehat{a}+\widehat{K} \otimes \widehat{a}^{\dagger} C \widehat{a}+\widehat{R} \otimes \widehat{a}^{\dagger}(f)+\widehat{R}^{\dagger} \otimes \widehat{a}\left(f^{*}\right)+\widehat{H}_{0} \otimes I,
$$

где область определения $\bar{A}$ совпадает с естественной областью определения выражения в правой части, $\widehat{a}^{\dagger}(f)$ и $\widehat{a}\left(f^{*}\right)$ - операторы рождения и уничтожения в $\mathscr{F}(L)$ соответственно (см. [1]), I - тождественный оператор в соответствующем пространстве. Тогда оператор $\bar{A}$ самосопряжен.

6. Теорема о представлении решений. Пусть $L$ - сепарабельное комплексное гильбертово пространство. Зафиксируем $b, f \in L$ и $t \geqslant 0$. Для $\varkappa=\rho, \alpha, \beta, \gamma, u, v$ определим меры $\widehat{\varkappa}_{t}$ на $\Sigma_{F}$ и их преобразования Фурье $\varkappa_{t}$ следующими формулами:

$$
\begin{gathered}
\hat{\rho}_{t}=\mu_{0, t}, \quad \rho_{t}: F^{\prime} \rightarrow \mathscr{L}(L), \quad \rho_{t}(x)=W_{0}^{t}(x) \\
\widehat{\alpha}_{t}=i f^{*} \int_{0}^{t} \mu_{0, \tau} * \nu_{\tau}^{\dagger} d \tau, \quad \alpha_{t}: F^{\prime} \rightarrow L, \quad \alpha_{t}(x)=i f^{*} \int_{0}^{t} R^{\dagger}(x(\tau)) W_{0}^{\tau}(x) d \tau ; \\
\widehat{\beta}_{t}=i \int_{0}^{t} \nu_{\tau} * \mu_{\tau, t} d \tau f, \quad \beta_{t}: F^{\prime} \rightarrow L, \quad \beta_{t}(x)=i \int_{0}^{t} W_{\tau}^{t}(x) R(x(\tau)) d \tau f ; \\
\widehat{\gamma}_{t}=i f^{*} \int_{0}^{t} d \tau \int_{0}^{\tau} d \sigma \nu_{\sigma} * \mu_{\sigma, \tau} * \nu_{\tau}^{\dagger} f+i \int_{0}^{t} \theta_{\tau} d \tau, \\
\gamma_{t}: F^{\prime} \rightarrow \mathbb{C}, \quad \gamma_{t}(x)=i f^{*} \int_{0}^{t} d \tau \int_{0}^{\tau} d \sigma R^{\dagger}(x(\tau)) W_{\sigma}^{\tau}(x) R(x(\sigma)) f+i \int_{0}^{t} H_{0}(x(\tau)) d \tau .
\end{gathered}
$$

Теорема 1. Пусть $K, R, R^{\dagger}, H_{0}: Q \times P \rightarrow \mathbb{C}$ - преобразования Фурье мер ограниченной вариачии $\mu, \nu, \nu^{\dagger}, \theta: \mathscr{B}(P \times Q) \rightarrow \mathbb{C}$ соответственно. Пусть $\widehat{K}, \widehat{R}, \widehat{R} \widehat{R}^{\dagger}, \widehat{H}_{0}:$ $L^{2}\left(\mathbb{R}^{l}\right) \rightarrow L^{2}\left(\mathbb{R}^{l}\right)$ - псевдодифференциальные операторы с qр-символами $K, R, R^{\dagger}, H_{0}$ соответственно. Предположим, что меры $\mu, \nu, \nu^{\dagger}$ и $\theta$ таковы, что оператор $\widehat{R}^{\dagger}$ является эрмитово сопряжсенным $к$ оператору $\widehat{R}$, а $\widehat{K}$ u $\widehat{H}_{0}-$ самосопряженные операторы.

Пусть Е и C-самосопряженные операторы в сепарабельном комплексном гильбертовом пространстве $L$ и $C$-ограниченный оператор.

Тогда для сильно непрерывной полугруппы $U_{t}=e^{i t \bar{A}}, t \geqslant 0$, г генератором $i \bar{A}$ справедливо равенство $\left(U_{t}(\psi \otimes e(b))\right)_{k}=I_{g}\left(\left(\rho_{t} b+\beta_{t}\right)^{\otimes k} e^{\alpha_{t} b+\gamma_{t}} / \sqrt{k !}\right) \psi$ для всех $\psi \in L^{2}\left(\mathbb{R}^{l}\right), b \in L u k=0,1,2, \ldots$. 
ЗАмЕчАниЕ 2 . Определим функции $g_{p q}, g_{w}: F \rightarrow \mathbb{R}$ так, что

$$
g_{p q}\left(y_{P}, y_{Q}\right)=\int_{0}^{\infty} y_{Q}[\tau, \infty) y_{P}(d \tau), \quad g_{w}\left(y_{P}, y_{Q}\right)=\frac{1}{2}\left(g\left(y_{P}, y_{Q}\right)+g_{p q}\left(y_{P}, y_{Q}\right)\right)
$$

Теорема 1 останется справедливой, если заменить функцию $g$ на $g_{w}$ (соответственно на $g_{p q}$ ) и считать, что функции $K, R, R^{\dagger}, H_{0}$ являются символами Вейля (соответственно $p q$-символами) операторов $\widehat{K}, \widehat{R}, \widehat{R}^{\dagger}, \widehat{H}_{0}$.

ЗАМЕчАнИЕ 3. Используя аналогичные конструкции и рассуждения, можно доказать аналог теоремы 1 для псевдодифференциальных операторов $\widehat{K}: L^{2}\left(\mathbb{R}^{l}\right) \rightarrow L^{2}\left(\mathbb{R}^{l}\right)$ вида

$$
(\widehat{K} \varphi)(q)=(2 \pi)^{-l} \iint K\left(q, q^{\prime}, p\right) e^{i p\left(q^{\prime}-q\right)} \varphi\left(q^{\prime}\right) d q^{\prime} d p, \quad \varphi \in L^{2}\left(\mathbb{R}^{l}\right),
$$

где $K(\cdot, \cdot, \cdot): Q \times Q \times P \rightarrow \mathbb{C}$ - символ оператора $\widehat{K}$.

Автор благодарит О.Г. Смолянова и А.М. Чеботарева за ряд полезных советов и стимулирующее внимание к работе.

\section{СПИСОК ЦИТИРОВАННОЙ ЛИТЕРАТУРЫ}

[1] Березин Ф. А. Метод вторичного квантования. М.: Наука, 1965.

[2] Данфорд Н., Шварц Дж. Линейные операторы. Общая теория. Т. 1. М.: ИЛ, 1962.

[3] Шефер Х. Топологические векторные пространства. М.: Мир, 1971.

[4] Смолянов О. Г., Шавгулидзе Е. Т. Континуальные интегралы. М.: МГУ, 1990.

[5] Смолянов О.Г. Бесконечномерные псевдодифференциальные операторы и квантование по Шредингеру // Докл. АН СССР. 1982. Т. 263. № 3. С. 558-561.

[6] Смолянов О.Г., Хренников А. Ю. Алгебра бесконечномерных псевдодифференциальных операторов // Докл. АН СССР. 1987. Т. 292. №6. С. 1310-1314.

[7] Smolyanov O.G., Weizsaecker H.v. Smooth probability measures and associated differential operators // Infinitely Dimensional Analysis. Quantum Probability and Related Topics. 1999. V. 2. № 1. P. 51-79. 